\title{
USE OF UAS FOR THE CONSERVATION OF HISTORICAL BUILDINGS IN CASE OF EMERGENCIES
}

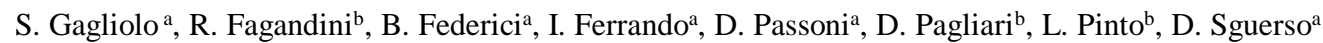 \\ ${ }^{a}$ Università degli Studi di Genova, DICCA - Laboratory of Geodesy, Geomatics and GIS, Via Montallegro, 1, 16145 Genoa \\ saragagliolo@hotmail.it, (bianca.federici,domenico.sguerso)@unige.it, ilaria.ferrando@edu.unige.it, \\ daniele.passoni@dicca.unige.it \\ b Politecnico di Milano, DICA - Geodesy and Geomatics section, Piazza L. da Vinci 32, 20133 Milan \\ (roberta.fagandini, diana.pagliari, livio.pinto)@ polimi.it
}

KEY WORDS: Emergency, Conservation, Safety, UAS Photogrammetry, Oblique Images, TLS

\begin{abstract}
:
The task of conservation and management of cultural heritage is quite central in Italy, which lists a high number of beautiful architectures. A quick and precise survey may be requested in case of calamity. In the present paper, the most commonly used survey techniques are discussed, focusing on their applications for the conservation of the artistic heritage in case of emergency. Particular attention is given to Unmanned Aerial Systems (UAS) photogrammetry and its potentiality in obtaining good results in terms of speed, cheapness, precision and accuracy, assuring at the same time the safety of the operators in critical situations (e.g. natural disasters). A case study, realized at the Castle of Casalbagliano (Alessandria, Italy), is discussed. Different image block configurations and acquisition geometries (nadiral and oblique images) have been exploited, with the aim of defining useful guidelines for emergencies UAS survey of partially collapsed structures. An application to a significative case study is introduced.
\end{abstract}

\section{INTRODUCTION}

The task of the conservation of cultural heritage has recently become very important, not only for documentation purposes, but also for management issues. A number of factors can modify the state of the heritage itself, such as natural disasters, wars or human negligence. For all these reasons, it is fundamental to create an appropriate digital documentation of the cultural heritage. In the last decades, a wide number of researchers focused their attention on cultural heritage documentation, realizing highly detailed 3D models. The purposes behind these researches could be very different, spacing from pure historical image documentation (Yastikli, 2007; Yilmaz, 2007), digital reconstruction of destroyed objects (Grün et al., 2004) or the creation of web-catalogues and virtual museum tours (Wojciechowski, 2004; Bruno et al., 2010). However, collecting documentary evidences can be useful also for historical building management as well as for damages quantification and restoration purposes. When an historical building menaces to collapse, the main goal to reach is the immediate securing of the site. This is necessary to: (1) ensure public security; (2) save the cultural value of the building itself; (3) remove the ruins of the already collapsed parts. In order to lead the securing operations, it is requested to know the current state of the structure; anyway, the critical situation (e.g. risk of collapse) prevents executing a standard survey. In these prohibitive conditions, the chosen survey technique should guarantee operator safety (e.g. avoid to approach the area), without compromising the quality of the data acquisition, resulting in an accurate and complete 3D model. In fact, it is fundamental to maintain precision and accuracy of the delivered product, guaranteeing at the same time quickness and cheapness.
A large number of sensors can be used in cultural heritage 3D mapping and modelling (see Remondino, 2011 for a complete review). Historically, Terrestrial Laser Scanners (TLSs) have been widely used for modelling purposes (Boehler, 2002; Fontana et al., 2003), while Unmanned Aerial Systems (UAS) represent a more recent and widespread technique. The use of UAS is quite common in architectural and cultural heritage from a plenty of applications (see for instance Themistocleous, 2015; Sauerbier and Eisenbeiss, 2010; Brutto et al., 2014) mainly because of its low cost and flexibility, combined with the possibility of surveying inaccessible areas. Nowadays, an open task is the use of these techniques in case of emergencies and postcalamitous event surveys, in order to document and quantify the damages, for helping during rescue operations and for the prevention of secondary collapses. Several authors have already discussed the use of UAS for post-seismic event and their integration with different surveying methods (see for instance Xu, 2014; Achille et al., 2015; Meyer et al., 2015; Ballarin et al., 2013).

The survey has to be planned accordingly, ensuring a quick data acquisition without compromising the quality of the final delivered 3D model. Especially in case of an extensive damaged area, it is important to consider the required accuracy level to recognize the main damages of the inaccessible structure.

Although in principle the strategies presented in this paper can be applied to any emergency, the focus of the analysis here discussed is the seismic occurrence. This work comes from the master thesis of one of the authors. Starting from the survey of the partially collapsed Castle of Casalbagliano (Alessandria, Italy), quite general criteria are discussed. They aim to be useful to plan, execute and elaborate UAS 
photogrammetric campaigns in case of emergencies. An analysis of the reachable accuracies is performed, realizing a comparison with TLS acquisitions. TLSs are commonly used in building inspections to realize 3D building models. However, they require to be stationed close to the inspected object. This represents a scenario that cannot be always possible in case of seismic events, without exposing operators to risks. Moreover, the TLS is incapable of acquiring data of the top of the roofs and requires multiple station positions to create a complete and reliable 3D model. Furthermore, TLSs are quite expensive. On the other hand, UAS allows to overfly the area and to obtain data using different acquisition geometry (e.g. nadiral and oblique images). For the same reasons, point clouds deriving from TLS scanning and UAS photogrammetry are often complementary from the geometrical point of view, while they represent a mutual term of comparison if both are present. The comparison between TLS and UAS surveys is discussed by means of a simulation of an earthquake scenario, with the main purpose of evaluating the feasibility of UAS photogrammetry for post-seismic and emergency surveying. For such purpose, the partially collapsed Castle of Casalbagliano has been used as a case study. Actually, the current situation is not due to any seismic event, but it could be reasonably assimilated to such scenario. The study area has been scanned using TLS, to produce a reference model. The building has been surveyed performing different UAS flights, considering different acquisition geometry. The guidelines derived from the case study have been consequently applied to the practical case of the Civic Tower and the Co-cathedral of Santa Maria Argentea of Norcia (Perugia, Italy), which were strongly damaged by the earthquake that affected Italy particularly in August and October 2016.

The paper is organized as follows. In Section 2, the selected test area and the data acquisition phases are presented. The photogrammetric data processing, the considered different scenarios and the computed results are discussed in Section 3. Section 4 deals with the comparison of the photogrammetric dense clouds and the laser scanner reference model, while in Section 5 the application of the Civic Tower and the Co-cathedral of Santa Maria Argentea of Norcia is illustrated. In Section 6, some final considerations about UAS photogrammetry for emergency scenario are discussed.

\section{THE CASE STUDY OF THE CASTLE OF CASALBAGLIANO}

\subsection{Selected test area}

The main purpose of this paper is to evaluate if UAS photogrammetry is suitable in case of post-seismic survey, defining the reachable accuracy level and some useful guidelines. The selected case study is the Castle of Casalbagliano (see Figure 1), located near Alessandria (Italy). The Castle of Casalbagliano was built at the end of XIII century. It is characterized by a square map, which is typical of medieval fortifications. It was originally built using bricks and it was restored several times, recently realizing some concrete structural enforcements. During World War I, it was used as a military hospital and then it was employed as fascist party headquarters. During the last decades, it suffered a progressive neglect and nowadays the structure is in poor conditions, with an effective risk of collapse. One of the reasons that brought the structure to the current state is the flood of Tanaro River, which affected Alessandria in 1994. Before the realization of the survey, a site inspection has been carried out, with the aim to define the optimal location of the station points used for the determination of the geodetic network and for the TLS acquisitions. The station points were chosen paying particular attention to guarantee their inter-visibility. Moreover, possible obstacles that could interfere with the UAS flight and with the Global Navigation Satellite System (GNSS) survey were observed and taken into account for the planning of the survey itself.

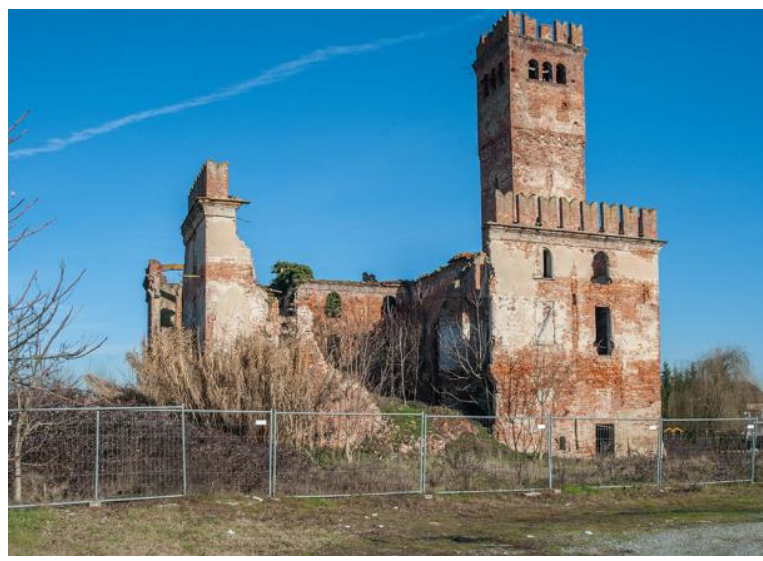

Figure 1. Castle of Casalbagliano.

\subsection{Data acquisition}

All the survey steps have been carefully planned and the date of the survey campaign was chosen paying particular attention to the weather (e.g. wind and rain can prevent its correct execution). The survey of the Castle of Casalbagliano took place in a single day, on $17^{\text {th }}$ March 2016.

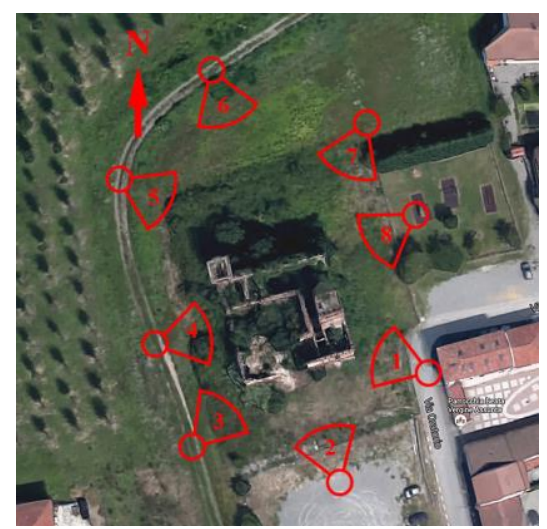

Figure 2. Locations of station points used for the laser scanner acquisition and for the determination of the geodetic network.

Eight station points were placed around the castle, at a reciprocal distance lower than $35 \mathrm{~m}$ (see Figure 2). Four points were set in correspondence of the corners of the castle perimeter, while the others were located near the middle points of each façade. The resulting geodetic network has 
been realized using a close transverse scheme and following a high redundant criterion (i.e. realizing measurements among all the station points that were reciprocally visible). The vertices of the geodetic network were used also as station points for the TLS acquisitions. The reference system was defined locally, using the first station point as the origin, the $\mathrm{z}$-axis oriented in the zenith direction and the $\mathrm{y}$ axis oriented parallel to one of the perimeter walls (i.e. towards the last point of the network). The $\mathrm{x}$-axis completes the right handed Cartesian reference frame.

The geodetic network has been measured using the Leica Nova MS60 MultiStation in Total Station (TS) mode. This instrument is characterized by an angular accuracy of $3 \mathrm{cc}$ and by a distance accuracy of $1 \mathrm{~mm}+1.5 \mathrm{ppm}$ with reflective prism. The measured network has been adjusted using the software provided by the instrument producer, resulting in a closing error of $50 \mathrm{cc}$. The average distance between the castle perimeter and the station points was of the order of 30-40 m, ensuring a sufficient high resolution in the resulting TLS point clouds. The terrestrial laser scanner acquisitions have been performed using a Z+F Imager ${ }^{\circledR}$ $5006 \mathrm{~h}$. With the aim to guarantee a spacing of about $1 \mathrm{~cm}$ in the acquired point clouds, the TLS scans have been realized using a resolution of 20,000 points $/ 360^{\circ}$. In order to align the different scans, 32 Forex reflective black and white targets have been arranged in the surveyed area. The targets have been placed on the castle façades and on different objects located around the scene (e.g. perimeter fences, trees etc.). Because of the scans have been acquired using a $360^{\circ}$ geometry, the targets have been distributed in any direction, ensuring a better results in the scans alignment phase. The coordinates of all the targets have been measured with the TS, ensuring the highest possible redundancy, and adjusted in the geodetic network.

The UAS survey has been carefully planned to guarantee the realization of a complete, accurate and precise final 3D model. For these reasons, two different flight configurations were considered, integrating complementary points of view. For the first configuration, a nadiral geometry has been used, while for the second one an oblique geometry has been evaluated. The former allows observing the building from the top, while the latter was used to acquire images of the perimeter walls. Some images have been acquired from the ground to avoid holes in the final 3D model. The UAS surveys have been realized using a Microcopter hexacopter, which is equipped by an on board low-cost GNSS receiver (Ublox LEA GH), a triaxial magnetometer and a Micro Electro-Mechanical Systems/ Inertial Measurement Unit (MEMS/IMU). The maximum payload is about $0.5 \mathrm{~kg}$ and it has a flight autonomy of about $15 \mathrm{~min}$. The images have been acquired using a Canon EOS-M camera, with a fixed focal length equal to $22 \mathrm{~mm}$. The average flight height for the nadiral flight was about $40 \mathrm{~m}$. A second flight with a nadiral configuration was realized over the tower, with a flight height of $50 \mathrm{~m}$. In both cases a high overlapping has been guaranteed (80\% along flight direction and $70 \%$ along cross direction). The resulting average Ground Sample Distance (GSD) was about $9 \mathrm{~mm}$. A third flight was accomplished with an oblique camera configuration, a flight height of about $50 \mathrm{~m}$ and describing a square along the building perimeter (maintaining a fixed distance of about 50 $\mathrm{m})$. The camera has been set up with a tilt angle of $45^{\circ}$, pointing at the centre of the described trajectory. The resulting overlapping was about $80 \%$. The photogrammetric block has been georeferenced using 19 Ground Control Points (GCPs), whose coordinates have been measured with both TS and GNSS (RTK mode, reaching $2 \mathrm{~cm}$ accuracy). The GCPs have been materialized on the ground using black and white square targets $(30 \mathrm{~cm}$ side) that have been spread out all over the test area. At the end of the survey campaign, all the station points were measured too with a GNSS receiver to georeference the final model in a global reference frame. The photogrammetric blocks have been adjusted using the coordinates estimated from the TS survey, with the aim to evaluate the quality of UAS photogrammetry alone, avoiding introducing error due to the GNSS survey.

\section{PHOTOGRAMMETIC DATA PROCESSING}

\subsection{Different processing features}

All the acquired images have been processed using the commercial software Agisoft Photoscan - version 1.2.6 (http://www.agisoft.com/), following the standard workflow proposed by the software itself. The hardware used for the photogrammetric processing is a HP Envy15 laptop (64 bits Windows 10 operating system, Intel ${ }^{\circledR}$ core $^{\mathrm{TM}}$ i7-550 CPU @ $240 \mathrm{GHz}$ processor and $16 \mathrm{~GB}$ of RAM, GeForce GTX $850 \mathrm{M}$ graphic card with $4096 \mathrm{MB}$ of dedicated memory).

After the recovering of the External Orientation (EO) parameters, the embedded 'optimize stage' was used (for all the considered scenarios) to perform the camera selfcalibration. In fact, it is quite important to refine the Internal Orientation (IO) parameters in case of blocks acquired from UAS. The parameters, estimated during a standard calibration procedure, could vary because of the impacts suffered by the lens during take-off and landings. The acquired images have been processed choosing different combinations of block configurations and constraints. The results have been evaluated in terms of final accuracy and computational cost. The first considered variable was the acquisition geometry. The images have been divided into three groups: nadiral images ( 83 frames), oblique images (61 frames) and terrestrial acquisitions (28 frames). A second variable taken into account was the number of GCPs. Three different constrain configurations were tested. In the first case the whole set of GCPs (19) was used, in the second scenario 12 GCPs have been used, while in the third scenario the minimum number of requested GCPs (3) was selected. The effects of the image resolution for both EO parameters estimation and dense point cloud generation have been evaluated too. Two different resolutions were analysed. The quality term 'high' corresponds to the use of images at their full resolution, while the term 'medium' corresponds to an image downscaling by a factor of 4 ( 2 time for each side).

\subsection{Different block configurations}

As already stressed before, several combination of processing parameters and block configurations have been analysed. Four combinations of the different acquisition geometries have been tested. In the first case, the combination of the images acquired during the nadiral flights and the oblique one $(\mathrm{N}+\mathrm{O})$ was evaluated. In the second case, the images of the nadiral flights were processed together with the ones acquired from the ground $(\mathrm{N}+\mathrm{T})$, while in the third case, all the acquired images have been used $(\mathrm{N}+\mathrm{O}+\mathrm{T})$. In the last case, the oblique 
photogrammetric block (O) has been processed separately. The solution composed only by images acquired during the nadiral flights was not created, because of the absence of images in correspondence of the perimeter walls, which would have resulted in an incomplete model. The combination of nadiral and terrestrial images $(\mathrm{N}+\mathrm{T})$ was discarded because the software was unable to orient the two dataset together. This behaviour is due to the limited overlapping among the nadiral images and the ones acquired from the ground. In fact, the former captured the castle from the top view, while the latter corresponded to the perimeters walls. Three different GCPs constraints have been considered for each of the already introduced block geometry. In particular, in the first scenario the whole set of 19 GCPs have been used. In the second case, only 12 GCPs have been used, and the remaining 7 points have been exploited as Check Points (CPs). Here, the GCPs have been selected with the aim to evaluate the worst possible scenario, such as the presence of heaps of rubble, which prevents to homogeneously distribute them. In the last scenario, the minimum number of GPCs requested for orienting a photogrammetric block (3) has been used, therefore the other 16 points have been considered as CPs. The distribution of GCPs and CPs is shown in Figure 3.

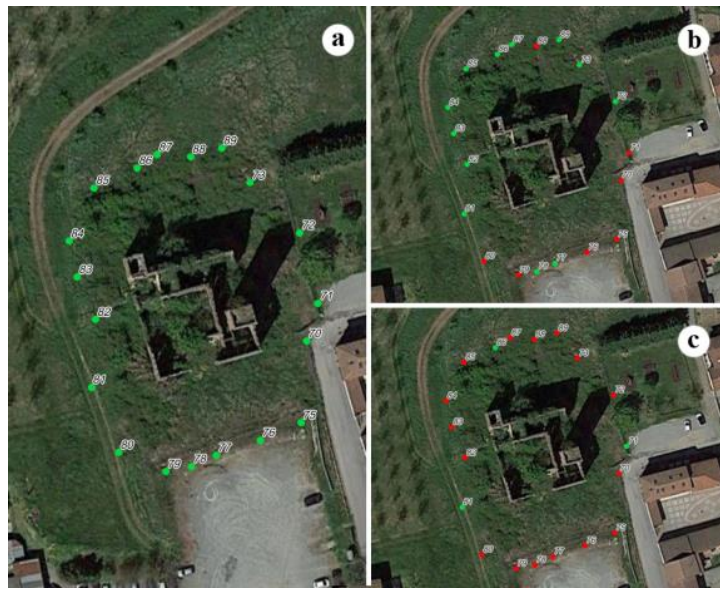

Figure 3. Locations of the GCPs and CPs over the surveyed area. GCPs are represented by green dots, while CPs by red dots. a) 19 GCPs case b) 12 GCPs case c) 3 GCPs case.

For each block geometry and constraint the influence of the image resolution has been considered too, by using different 'alignment' quality values of the Photoscan software. The quality of the computed solution has been evaluated by considering the Root Mean Square Errors (RMSE) of the CPs. Due to the absence of CPs for the 19 GCPs case, the quality of the block has been evaluated by computing the RMSE among the GCPs coordinates and the ones estimated using the computed EO solution. The results are shown in Table 1, suggesting some interesting considerations.

\begin{tabular}{|c|c|c|c|c|}
\hline $\begin{array}{c}\text { Block } \\
\text { geometry }\end{array}$ & \# GCP & \# CP & $\begin{array}{c}\text { Image } \\
\text { quality }\end{array}$ & $\begin{array}{c}\text { RMSE } \\
{[\mathrm{m}]}\end{array}$ \\
\hline $\mathrm{O}$ & 19 & 0 & High & 0.023 \\
\hline $\mathrm{O}$ & 19 & 0 & Medium & 0.023 \\
\hline $\mathrm{O}$ & 12 & 7 & High & 0.037 \\
\hline $\mathrm{O}$ & 12 & 7 & Medium & 0.037 \\
\hline $\mathrm{O}$ & 3 & 16 & High & 0.029 \\
\hline
\end{tabular}

\begin{tabular}{|c|c|c|c|c|}
\hline $\mathrm{O}$ & 3 & 16 & Medium & 0.029 \\
\hline $\mathrm{N}+\mathrm{O}$ & 19 & 0 & High & 0.023 \\
\hline $\mathrm{N}+\mathrm{O}$ & 19 & 0 & Medium & 0.023 \\
\hline $\mathrm{N}+\mathrm{O}$ & 12 & 7 & High & 0.038 \\
\hline $\mathrm{N}+\mathrm{O}$ & 12 & 7 & Medium & 0.037 \\
\hline $\mathrm{N}+\mathrm{O}$ & 3 & 16 & High & 0.030 \\
\hline $\mathrm{N}+\mathrm{O}$ & 3 & 16 & Medium & 0.030 \\
\hline $\mathrm{N}+\mathrm{O}+\mathrm{T}$ & 19 & 0 & High & 0.023 \\
\hline $\mathrm{N}+\mathrm{O}+\mathrm{T}$ & 19 & 0 & Medium & 0.023 \\
\hline $\mathrm{N}+\mathrm{O}+\mathrm{T}$ & 12 & 7 & High & 0.037 \\
\hline $\mathrm{N}+\mathrm{O}+\mathrm{T}$ & 12 & 7 & Medium & 0.038 \\
\hline $\mathrm{N}+\mathrm{O}+\mathrm{T}$ & 3 & 16 & High & 0.030 \\
\hline $\mathrm{N}+\mathrm{O}+\mathrm{T}$ & 3 & 16 & Medium & 0.030 \\
\hline
\end{tabular}

Table 1. RMSE of the considered scenarios.

First of all, there is no impact of the quality 'alignment' parameters (i.e. the resolution of the images used during the tie points search). This issue cannot be considered as a general behavior, because the impact of the image quality is strictly connected to the image texturing. However, the authors are quite confident to suggest that for emergencies applications the choice of the medium quality will not affect the results. It could be a proper choice in case a quicker solution is needed, because it allows speeding up the EO parameters computation of about $30 \%$.

The requested time to complete the tie points extraction and matching and the EO parameters computation is strictly connected to the number of images that compose the photogrammetric block and to the availability of flight path information (position and altitude), which helps in the image pair pre-selection. For all the analyzed scenarios, these data were not available. The longest processing time has been registered for the $\mathrm{N}+\mathrm{O}+\mathrm{T}$ case. Nevertheless, it was lower than 1 hour. Additional 2-3 hours were requested for dense cloud generation.

The second consideration is that the acquisition geometry of the photogrammetric block did not influence the results, mainly because each single photogrammetric block was already auto-consistent and has been acquired following a rigorous planning, guaranteeing a high overlapping and the completeness of the final model.

The RMSE are below $0.03 \mathrm{~m}$ for all acquisition geometry for both 19 GCPs and 3 GCPs cases. It slightly increases in case of the 12 GCPs scenario, mainly because of the bad distribution of the GCPs themselves. However, this case was considered for simulating a critical situation (e.g. operator's difficulties in measuring GCPs because of the presence of ruins) and the results show that even in this case the influence on the quality of the photogrammetric block is minimal, and in line with the accuracy requested in case of an emergency survey.

A dense cloud has been generated for each photogrammetric block, using a medium resolution (see Figure 4). 


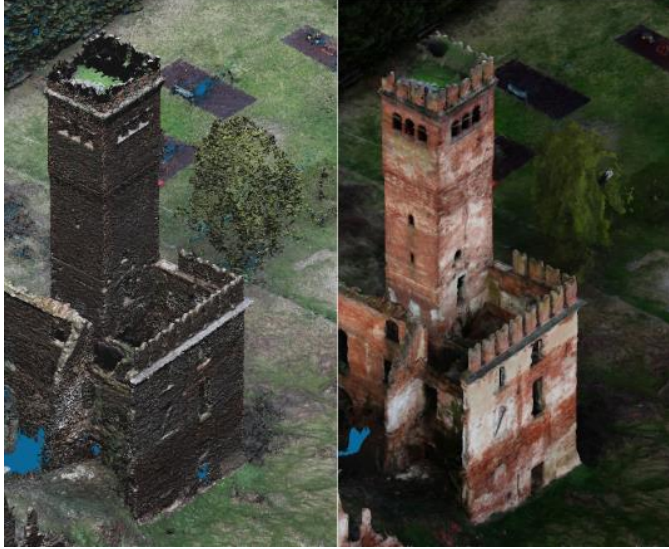

Figure 4. An example of photogrammetric dense point cloud (left) and the correspondent textured meshed model (right).

With the aim to evaluate the spacing of the generated point clouds, the mean distance between each point and the corresponding nearest neighbor was computed. The average distance between points was equal to $0.025 \mathrm{~m}$, with a standard deviation of $0.012 \mathrm{~m}$. For the oblique image case, the point cloud has been created also using a high density, resulting in a thicker spacing $(0.018 \mathrm{~m}$ with a standard deviation of 0.009).

\section{COMPARISON WITH LASER SCANNER POINT CLOUD}

\subsection{Laser scanner data processing}

The 3D reference model has been created by means of registration of the 8 laser scanner acquisitions. The point clouds have been aligned using the $\mathrm{Z}+\mathrm{F}$ LaserControl software, detecting on the different scans the 32 reflecting targets. In order to enforce the alignment solution, a number of easily identifiable corresponding natural points has been exploited too. As a result, it was possible to identify an average of 10 corresponding observations for each pair of scans. The different scans have been registered through a least square adjustment procedure resulting in a single cloud, characterized by estimated standard deviation of 2.2 $\mathrm{mm}$ and a mean equal to $6.8 \mathrm{~mm}$. In Table 2, the statistics computed after the alignment procedure are shown. The obtained results are coherent with the wished level of accuracy $(0.01 \mathrm{~m})$.

\begin{tabular}{|c|c|}
\hline Number of reflective targets & 32 \\
\hline Number of natural targets & 28 \\
\hline $\begin{array}{c}\text { Average number of } \\
\text { corresponding points }\end{array}$ & 10 \\
\hline Mean [mm] & 6.8 \\
\hline Standard deviation [mm] & 2.2 \\
\hline Maximum deviation [mm] & 11.3 \\
\hline
\end{tabular}

Table 2. TLS point clouds alignment statistics.

Because the coordinates of the reflective targets were measured with the TS, the final 3D model is expressed in the defined local reference system, which is the same used for the photogrammetric image processing.
The different point clouds have been manually edited and merged in a single 3D model, using the open source software CloudCompare (CC) - version 2.8.1 (http://www.cloudcompare.org). The final merged model was subsampled with the same software. The chosen subsample distance was equal to $0.011 \mathrm{~m}$, corresponding to a filter of about $50 \%$ of the scanned points. This operation was useful to better manage the final model; in fact, the number of points was reduced from 596255 to 307487 , without compromising its accuracy.

\subsection{Comparison between the photogrammetric and the laser scanner point clouds}

The photogrammetric dense clouds have been compared with the reference laser scanner point cloud, for each one of the evaluated scenarios. The comparisons have been performed using the Multiscale Model to Model Cloud Comparison (M3C2) algorithm (Lague et al., 2013), embedded within the CC software. Because of the abovementioned considerations about the photogrammetric solutions, the $\mathrm{M} 3 \mathrm{C} 2$ comparisons are presented and discussed only for the most representative cases. In particular, the different block geometry configurations with 19 GCPs have been considered. Moreover, for the N+O case, the analyses have been carried out also considering different block constrains (19, 12 or 3 GCPs). Then, an additional analysis has been performed considering only the tower of the Castle (extracted from the N+O+ T 19 GCPs case), which is the better conserved part, with a minimum presence of bushes.

In Table 3, the statistics about the distances computed with the $\mathrm{M} 3 \mathrm{C} 2$ algorithm are shown. These values have been obtained excluding the extreme values $(7.5 \%$ right and left tails of the absolute frequency distribution) that could be assimilated to outliers due to the presence of vegetation. The results obtained by comparing the photogrammetric and the reference TLS datasets confirm some of the trends already discussed about the photogrammetric block precision (see Section 3.2). The oblique geometry scenario represents the best solution. In fact, it is characterized by the lower RMS $(0.032 \mathrm{~m})$, computed considering the distances between the photogrammetric and the laser scanner point clouds. The quality of the photogrammetric dense clouds slightly worsens adding images characterized by different acquisition geometries to the processing. In fact, for the $\mathrm{N}+\mathrm{O}$ case, the RMS is equal to $0.048 \mathrm{~m}$, while it becomes equal to $0.059 \mathrm{~m}$ for the $\mathrm{N}+\mathrm{O}+\mathrm{T}$ scenario. In Figure 5, a graphic representation of the $\mathrm{M} 3 \mathrm{C} 2$ performed analysis is shown.

\begin{tabular}{|c|c|c|c|c|c|c|}
\hline case & $\begin{array}{c}\# \\
\text { GCP }\end{array}$ & $\begin{array}{c}\text { Mean } \\
{[\mathrm{m}]}\end{array}$ & $\begin{array}{c}\text { Std } \\
{[\mathrm{m}]}\end{array}$ & $\begin{array}{c}\text { Min } \\
{[\mathrm{m}]}\end{array}$ & $\begin{array}{c}\text { Max } \\
{[\mathrm{m}]}\end{array}$ & $\begin{array}{c}\text { RMS } \\
{[\mathrm{m}]}\end{array}$ \\
\hline $\mathrm{N}+\mathrm{O}+\mathrm{T}$ & 19 & 0.010 & 0.058 & -0.197 & 0.393 & 0.059 \\
\hline $\mathrm{N}+\mathrm{O}$ & 19 & 0.006 & 0.048 & -0.156 & 0.314 & 0.048 \\
\hline $\mathrm{O}$ & 19 & 0.005 & 0.032 & -0.225 & 0.118 & 0.032 \\
\hline $\mathrm{N}+\mathrm{O}$ & 12 & -0.008 & 0.078 & -0.572 & 0.158 & 0.079 \\
\hline $\mathrm{N}+\mathrm{O}$ & 3 & 0.007 & 0.045 & -0.115 & 0.317 & 0.046 \\
\hline Tower & 19 & -0.001 & 0.033 & -0.184 & 0.148 & 0.033 \\
\hline
\end{tabular}

Table 3. Statistics about the M3C2 comparison performed between the different photogrammetric point clouds and the reference TLS one. 


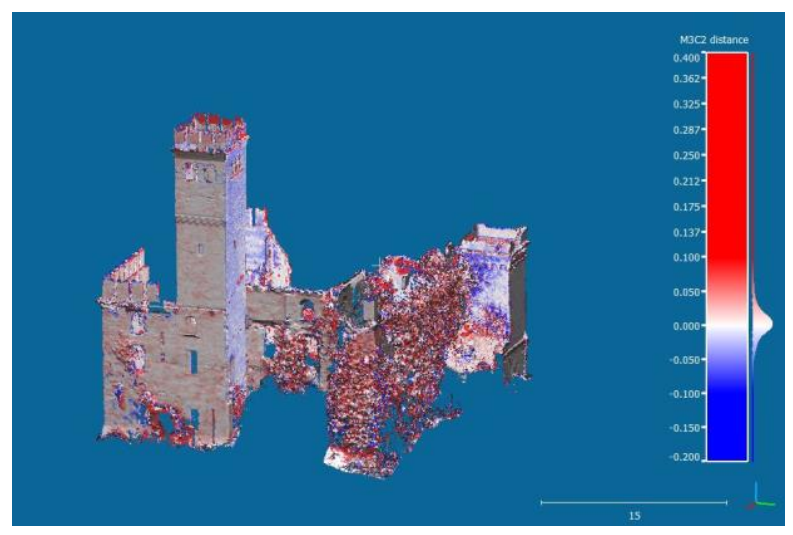

Figure 5. Distances (in meters) between the photogrammetric dense cloud ( $\mathrm{N}+\mathrm{O}+\mathrm{T}$ case) and the reference TLS point cloud, computed using the $\mathrm{M} 3 \mathrm{C} 2$ algorithm for the entire castle.

The first consideration that emerges from the statistics shown in Table 3 is that the best results can be obtained using an homogenous dataset, in terms of geometry acquisition. The introduction of nadiral and terrestrial images implicates more redundancy, resulting in more noisy dense clouds. During the photogrammetric processing, a single camera self-calibration was performed, meaning that the acquisition conditions were assumed fixed among the different surveys. Considering different block constrains, it is quite evident that the 19 and 3 GCPs solutions are characterized by the same level of errors, while the RMS increased for the 12 GCPs case. These results confirmed the ones previously discussed about the quality of the photogrammetric solutions (see Table 1). The abovementioned worsening could be mainly ascribed to the bad quality of the GCPs distribution in the last case. The accuracy obtained using the minimum constrain solution (3 GCPs) are comparable with the one that characterize the 19 GCPs case, meaning that in case of emergencies could be sufficient to use a low number of well distributed GCPs, for a preliminary quick solution. However, it is recommended to use at least few more GPSs to allow the identification of possible outliers.

The $\mathrm{M} 3 \mathrm{C} 2$ algorithm analysis has been performed also considering a portion of the model corresponding to tower of the castle. This area has been selected because it represents a building part that is less damaged and not overwhelmed by bushes. In this case, the computed differences are less spread and the distribution of the residual is quite symmetric (see Figure 6), with an overall RMS equal to $0.033 \mathrm{~m}$.

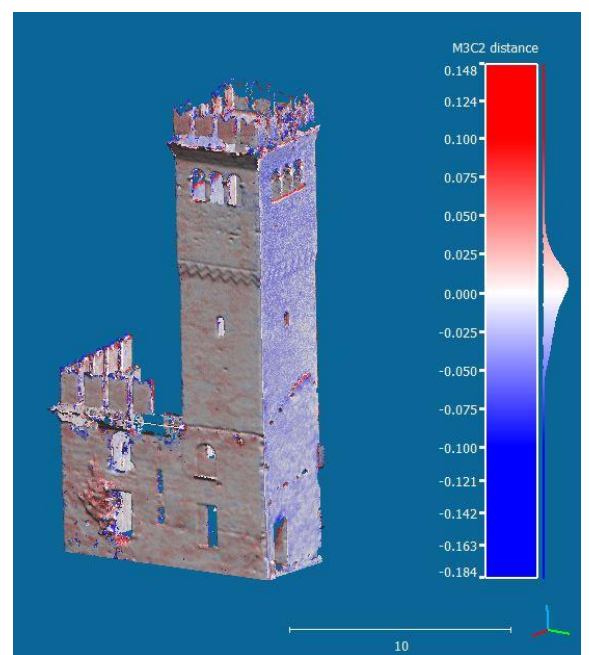

Figure 6. Distances (in meters) between the photogrammetric dense cloud $(\mathrm{N}+\mathrm{O}+\mathrm{T}$ case $)$ and the reference TLS point cloud, computed using the $\mathrm{M} 3 \mathrm{C} 2$ algorithm for the tower of the castle.

\section{THE APPLICATION TO NORCIA CASE STUDY}

As known, Central Italy has been hit by several and tragic seismic events since August 2016, that have considerably damaged or destroyed the cultural and historical heritage.

To prevent the risk of collapse of the remaining structures in case of future aftershocks, and to support the planning for securing the sites, a quick and accurate $3 \mathrm{D}$ survey is mandatory.

Therefore, the criteria and guidelines described in the present paper have been applied to the practical case of Norcia, resulting extremely useful to survey the area from the proper safe distance, guaranteeing at the same time good precision and accuracy standards (Figure 7).

Gter Ltd Innovation in Geomatics, Gnss and Gis, spin-off of the Genoa University, has surveyed the Civic Tower and the Co-cathedral of Santa Maria Argentea on behalf of the Italian Ministry of Cultural Heritage and Activities and Tourism (MiBACT).

The UAS surveys have been realized using a DJI INSPIRE 1 , which is characterized by a maximum payload of about $0.600 \mathrm{~kg}$ and a flight autonomy of about $18 \mathrm{~min}$. The images have been acquired using a Zenmuse X5 camera, with a fixed focal length equal to $15 \mathrm{~mm}$.

Despite the guidelines derived from the analysis previously discussed on the Castle of Casalbagliano case study, from which that the oblique flight seems sufficient to obtain good results in point cloud generation, in this case it was necessary to accomplish a second flight with a nadiral configuration. The top view acquisition has allowed having a better overlapping in the central part of the Co-cathedral, avoiding the lack of data due to the presence of obstacles or shadows.

The flight has been accomplished with an oblique camera configuration, with a flight height of about $50 \mathrm{~m}$ and describing a square along the building perimeter (maintaining a fixed distance of about $50 \mathrm{~m}$ ). The camera has been set up with a tilt angle of $45^{\circ}$, pointing at the centre of the described trajectory. 


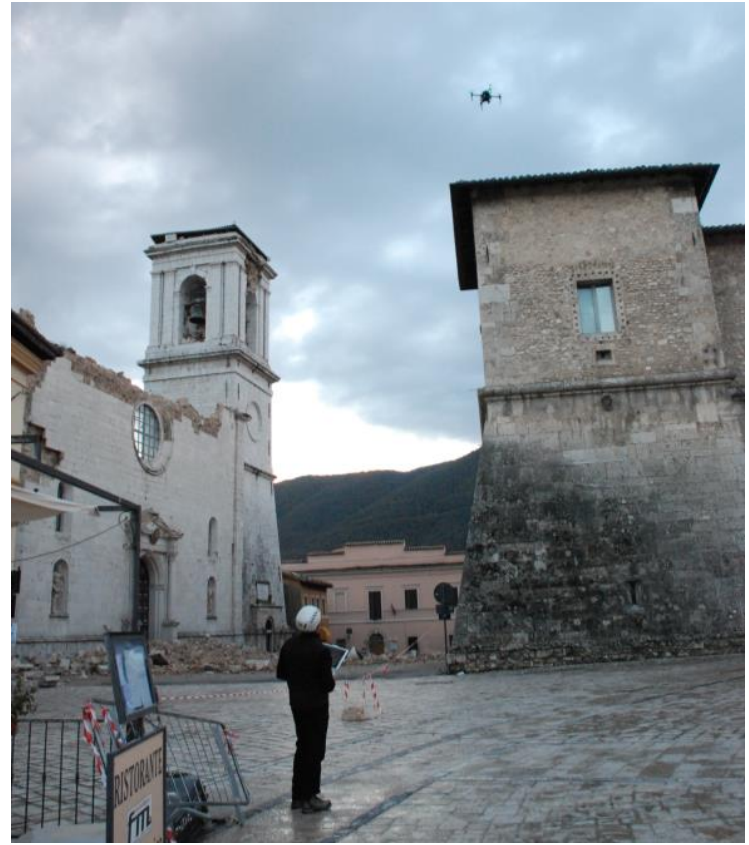

Figure 7. Photogrammetric survey with UAS.

The photogrammetric survey has been processed using the commercial software Agisoft Photoscan (version 1.2.6) to produce dense clouds, which were used to retrieve measures and geometrical information about the various structural elements of the Tower and the Co-cathedral. Therefore, it was possible to plan the provisional reinforcement systems, which were installed the day after the delivering of dense point clouds (i.e. the day after the survey itself). In this case, also 3D textured models and orthophotos of the horizontal and vertical planes have been realized to create a proper documentation, useful for the preservation of cultural and historical heritage. Moreover, the 3D model can be used for further studies such as the analyses of structures in response to other earthquakes. These 3D models can accurately describe the constructions with an appropriate level of detail for Finite Element Method (FEM) for structural analysis.

Gter Ltd has made available its web service 3DHosting (http://www.gter.it/3dhosting/) for the publication of 3D models and point clouds. The service is based on the Open Source software package 3DHOP 4.0 (http://vcg.isti.cnr.it/3dhop/) for the publication of highresolution 3D models and on Potree 1.5RC (http://potree.org/), a WebGL viewer, for the publication of large point clouds. 3DHosting allows sharing data with all the involved operators, providing tools for models navigation, query, measuring and creation of sections (Figure 8).

This and other later experiences gained in the context of emergencies survey (which will be object of dedicated works) have demonstrated the proper way of operating and processing, with the reliability of the obtained results at the service of structural engineering.
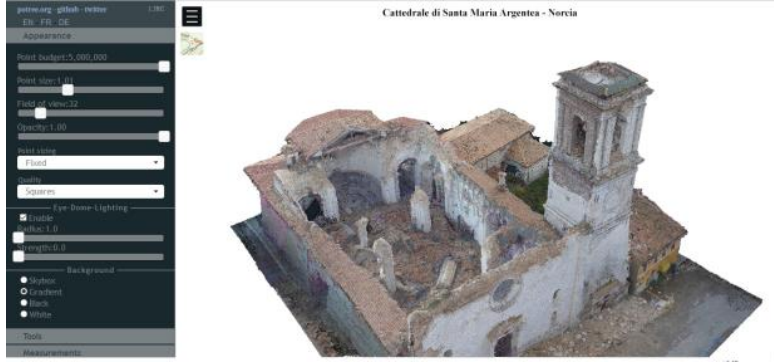

Figure 8. Point cloud of the Co-cathedral of Santa Maria Argentea deriving from photogrammetric UAS survey.

\section{CONCLUSIONS}

In this work, the use of UAS photogrammetry for emergency survey of collapsed structures in case of seismic event has been discussed, focusing on the impact of different block geometry configurations (nadiral, oblique and terrestrial acquisitions). The impact of the distribution of the GCPs and processing parameters (i.e. image resolution during tie points search and matching) has been analysed too. The building selected for the case study here discussed is the Casalbagliano Castle, which consists in a set of ruins, swarmed by vegetation.

The quality of the different photogrammetric solutions has been evaluated by means of TLS 3D model. The resolution of the reference model was of about $0.01 \mathrm{~m}$, which is comparable to precision request to identify the most important structural cracks. The guidelines extrapolated from the performed analysis have been used for the practical case study of the Civic Tower and Co-cathedral of Santa Maria Argentea of Norcia, which were strongly damaged because of the earthquake that affected Italy particularly in August and October 2016.

The quality of the photogrammetric solutions was evaluated twice, using two independent approaches. First, the residual of the checkpoints has been analysed and used to define the quality of the different cases. A second analysis has been carried out by comparing each dense photogrammetric point cloud with the reference TLS point cloud, applying the $\mathrm{M} 3 \mathrm{C} 2$ algorithm. A number of interesting considerations have emerged from the performed tests. The best block configuration is the one with the oblique geometry, considered as an independent dataset. This geometry ensured a complete reconstruction of the investigated buildings (both the perimeter walls and the top parts of the castle). Considering a pure photogrammetric point of view, this geometry was characterized by a block quality of 0.023 $\mathrm{m}$, evaluated in terms of RMSE between the GCPs coordinates and the estimated ones. This accuracy is in line with the results obtained adding also nadiral and terrestrial images, suggesting that there is no significant improvement in combining blocks acquired using different geometry, if the single block is already auto-consistent. On the other hand, the effect of such operation is the increase of the computation time, which could be a key factor in case of emergencies processing. The quality of the oblique solution is confirmed by the comparison performed using the TLS reference model. The delivered photogrammetric point clouds have been compared with the TLS scan by means of the $\mathrm{M} 3 \mathrm{C} 2$ algorithm, which computes the distance from the reference solution, for each point of the point cloud. The oblique geometry case represents the best solution in terms 
of M3C2 comparison, with an overall RMS in the order of $0.03 \mathrm{~m}$. In fact, the adding of images acquired using in different geometries (nadiral flights and terrestrial frames) worsens the quality of the produced dense clouds. This suggests that could be too simplistic to consider a single self-calibration; better results could be obtained by performing different camera self-calibration for each geometry acquisition.

The GCPs distribution affected the quality of the photogrammetric solution (and consequently of the generated dense clouds) if they are not well distributed over the surveyed area. This scenario was considered with the aim to simulate the impossibility of safely reaching a portion of the investigated area because of ruins or unstable part of the building. However, the use of the minimum constrain solution ( 3 GCPs) has a negligible impact on the final model accuracy. These results suggest that in case of emergencies a low number of well distributed GCPs could be sufficient, minimizing the time request for the on-site surveying operations.

\section{ACKNOWLEDGEMENTS}

The authors would like to thank for the provided support:

- Comune di Alessandria, in particular Arch. Marco Genovese and Geom. Gianfranco Ferraris, for their availability;

- Soprintendenza belle arti e paesaggio della Provincia di Alessandria, in particular Arch. Luigi Pedrini and Dott. Valentina Uras;

- MiBACT, Italian Ministry of Cultural Heritage and Activities and Tourism;

- Gter Ltd Innovation in Geomatics, Gnss and Gis, spin-off of the Genoa University for its availability;

- The co-supervisors of the thesis of one of the authors, Eng. Serena Cattari and Arch. Rita Vecchiattini, respectively at DICCA and DAD Departments at the Genoa University.

\section{REFERENCES}

Achille, C., Adami, A., Chiarini, S., Cremonesi, S., Fassi, F., Fregonese, L., Taffurelli, L., 2015. UAV-based photogrammetry and integrated technologies for architectural applications-Methodological strategies for the after-quake survey of vertical structures in Mantua (Italy). Sensors 15(7), pp. 15520-15539.

Ballarin, M., Buttolo, V., Guerra, F., Vernier, P., 2013. Integrated surveying techniques for sensitive areas: San Felice sul Panaro. ISPRS Annals of the Photogrammetry, Remote Sensing and Spatial Information Sciences 5, W1.

Boehler, W., Heinz, G., Marbs, A., 2002. The potential of non-contact close range laser scanners for cultural heritage recording. International Archives of Photogrammetry Remote Sensing and Spatial Information Sciences 34(5/C7), pp. $430-436$.

Bruno, F., Bruno, S., De Sensi, G., Luchi, M. L., Mancuso, S., Muzzupappa, M., 2010. From 3D reconstruction to virtual reality: A complete methodology for digital archaeological exhibition. Journal of Cultural Heritage 11(1), pp. 42-49.

Brutto, M. L., Garraffa, A., Meli, P., 2014. UAV platforms for cultural heritage survey: first results. ISPRS Annals of the Photogrammetry, Remote Sensing and Spatial Information Sciences 2(5), p. 227.

Fontana, R., Gianfrate, G., Greco, M., Marras, L., Materazzi, M., Pampaloni, E., Pezzati, L., 2003. Time of flight laser scanner for architectural and archaeological applications. Proceedings of SPIE, Vol. 5146, pp. 185-193.

Grün, A., Remondino, F., and Zhang, L., 2004. Photogrammetric reconstruction of the great Buddha of Bamiyan, Afghanistan. The Photogrammetric Record 19.107, pp. 177-199.

Lague, D., Brodu, N., Leroux, J., 2013. Accurate 3D comparison of complex topography with terrestrial laser scanner: application to the Rangitikei canyon (N-Z). ISPRS Journal of Photogrammetry and Remote Sensing 82, pp. 1026

Meyer, D., Hess, M., Lo, E., Wittich, C. E., Hutchinson, T. C., Kuester, F., 2015. UAV-based post disaster assessment of cultural heritage sites following the 2014 South Napa Earthquake. Digital Heritage, Vol. 2, pp. 421-424.

Remondino, F., 2011. Heritage recording and 3D modeling with photogrammetry and 3D scanning. Remote Sensing 3(6), pp. 1104-1138.

Sauerbier, M., Eisenbeiss, H., 2010. UAVs for the documentation of archaeological excavations. International Archives of Photogrammetry, Remote Sensing and Spatial Information Sciences 38 (Part 5), pp. 526-531.

Themistocleous, K., Ioannides, M., Agapiou, A., Hadjimitsis, D. G., 2015. The methodology of documenting cultural heritage sites using photogrammetry, UAV, and 3D printing techniques: the case study of Asinou Church in Cyprus. In Third International Conference on Remote Sensing and Geoinformation of the Environment, pp. 953510-953510.

Wojciechowski, R., Walczak, K., White, M., Cellary, W., 2004. Building virtual and augmented reality museum exhibitions. Proceedings of the ninth international conference on 3D Web technology, ACM.

Xu, Z., Yang, J., Peng, C., Wu, Y., Jiang, X., Li, R., Zeng, Y., Gao, Y., Liu, S., Tian, B., 2014. Development of an UAS for post-earthquake disaster surveying and its application in Ms7. 0 Lushan Earthquake, Sichuan, China. Computers \& Geosciences 68, pp. 22-30.

Yastikli, N., 2007. Documentation of cultural heritage using digital photogrammetry and laser scanning. Journal of Cultural Heritage 8.4, pp. 423-427.

Yilmaz, H. M., Yakar, M., Gulec, S. A., Dulgerler, O. N., 2007. Importance of digital close-range photogrammetry in documentation of cultural heritage. Journal of Cultural Heritage 8.4, pp. 428-433. 